\title{
Panoramic Visualization of Lithiation of Copper Sulfide by In Situ STEM
}

\author{
Kai $\mathrm{He}^{1,2, *}$, Sungkyu Kim ${ }^{1,2}$, Sooyeon Hwang ${ }^{3}$, Dong $\mathrm{Su}^{3}$
}

${ }^{1}$ Department of Materials Science and Engineering, Clemson University, Clemson, SC 29634, USA

2. Department of Materials Science and Engineering, Northwestern University, Evanston, IL 60208, USA

${ }^{3 .}$ Center for Functional Nanomaterials, Brookhaven National Laboratory, Upton, NY 11973, USA

Two-dimensional (2D) transition metal chalcogenides have been recently used as electrode materials for lithium ion batteries because they usually have open space in between their unique layered structures to accommodate reversible lithium insertion [1]. From electrochemistry perspective, these groups of materials are considered to undertake conversion reactions in addition to the lithium intercalation process. Despite extensive studies on performance optimization, mechanistic understanding of the phase transformations during lithiation of these materials is of great scientific interest. The rapid advancement of in-situ electron microscopy allows us to conduct dynamic observations of structure change in real time. It is noted that lithiation process may show large variations with respect to the electrode material's geometry, orientation, and size distributions; and thus a large number of statistical observations are required to reveal the true nature of such reactions [2], which may not be applicable in some circumstances.

Here, we use in-situ scanning transmission electron microscopy (STEM) method to study the structural, morphological, and chemical evolutions in individual copper sulfide $(\mathrm{CuS})$ nanoflakes during lithiation process [3]. The $\mathrm{CuS}$ nanoflake is an ideal model because it has the regular in-plane geometry of hexagon and the ultrathin thickness, as shown in Figure 1a. The high-resolution TEM images of the base plane and the side view (Figure 1a) indicate the interlayer channels for lithium insertion. This unique geometry determines the difference of lithiation between plan-view and side-view. However, we aim to achieve the panoramic visualization of the lithiation process in both viewing directions from a single observation. As shown in Figure 1b, a few nanoflakes were placed in close vicinity, so that they should be subject to the highly similar electrochemical environment. Using in-situ STEM HAADF imaging, we were able to capture the evolution of lithiation in both plan-view (marked by green dashed box) and side-view (marked by yellow dashed box). By analyzing the time-sequenced snapshots, we obtained the detailed information of plan-view (Figure 1c) and side-view (Figure 1d) lithiation processes, which explicitly revealed the two-step lithiation pathway through $\mathrm{Li}$ intercalation and $\mathrm{Cu}$ extrusion, respectively, which can be overlapped or in the sequential order depending on the localized kinetic effect. We further clarified that the phase transformation is highly kinetically-driven, in which lithium ions rapidly intercalate into the 2D van der Waals-stacked interlayers in the initial stage, and further lithiation induces the $\mathrm{Cu}$ extrusion via a displacement reaction mechanism rather than the typical conversion reactions. Our findings obtained from $\mathrm{CuS}$ nanoflakes shed light on the mechanistic understanding of nanoscale lithiation in other 2D transition metal chalcogenide systems. The experimental strategy of one-shot panoramic visualization is useful and applicable to many other materials that have geometry-dependent reaction propagations [4].

\section{References:}

[1] L Peng, et al, Adv. Energy Mater. 6 (2016) 1600025.

[2] K He, et al, Nano Lett. 15, (2015) 1437. 
[3] K He, et al, Nano Lett. 17, (2017) 5726.

[4] This work made use of microscopy facilities at Brookhaven National Laboratory supported by U.S. Department of Energy, Office of Basic Energy Sciences, under Contract Number DE-SC-00112704; and the EPIC facility of Northwestern University's NUANCE Center, which has received support from the Soft and Hybrid Nanotechnology Experimental (SHyNE) Resource (NSF ECCS-1542205); the MRSEC program (NSF DMR-1121262) at the Materials Research Center.
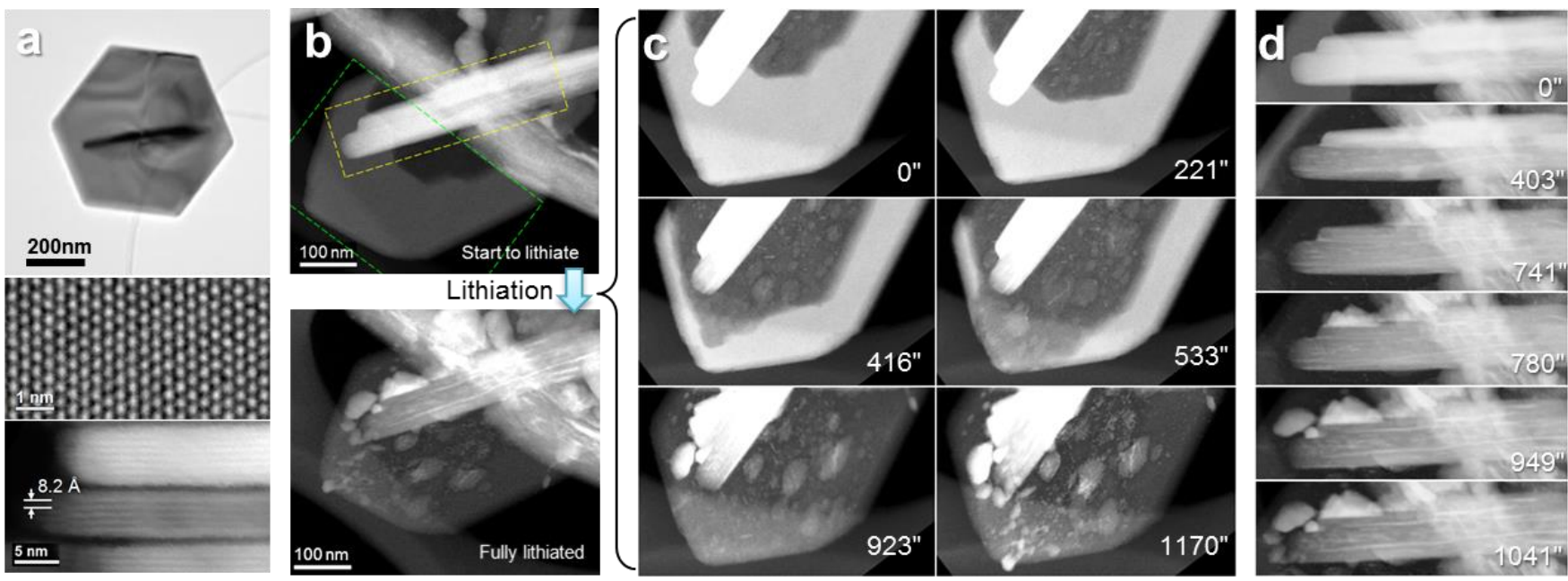

Figure 1. (a) TEM images showing morphology and atomic structures of the pristine CuS nanoflake. (b) HAADF-STEM images showing CuS nanoflakes before and after lithiation reaction. In-situ STEM snapshots showing lithiation process along (c) plan-view and (d) side-view, respectively. 